\title{
Artificial Neural Networks and Human Brain: Survey of Improvement Possibilities of Learning
}

\author{
Aleksejs Zorins, Peter Grabusts \\ Rezeknes Augstskola, Faculty of Engineering, \\ Atbrivosanas aleja 115, Rezekne, LV-4601, Latvia, \\ E-mail: Aleksejs.Zorins@ru.lv,Peteris.Grabusts@ru.lv
}

\begin{abstract}
There are numerous applications of Artificial Neural Networks (ANN) at the present time and there are different learning algorithms, topologies, hybrid methods etc. It is strongly believed that ANN is built using human brain's functioning principles but still ANN is very primitive and tricky way for real problem solving. In the recent years modern neurophysiology advanced to a big extent in understanding human brain functions and structure, however, there is a lack of this knowledge application to real ANN learning algorithms. Each learning algorithm and each network topology should be carefully developed to solve more or less complex problem in real life. One may say that almost each serious application requires its own network topology, algorithm and data pre-processing. This article presents a survey of several ways to improve ANN learning possibilities according to human brain structure and functioning, especially one example of this concept - neuroplasticity - automatic adaptation of ANN topology to problem domain.
\end{abstract}

Keywords: Artificial Neural Networks, Brain Networks, Artificial Neural Network Learning Algorithms.

\section{INTRODUCTION}

There are numerous applications of Artificial Neural Networks (ANN) at the present time and there are different learning algorithms, topologies, hybrid methods etc. It is strongly believed that ANN is built using human brain's functioning principles but still ANN is very primitive and tricky way for real problem solving, because in any application should be found answers to the following questions [1]:

- Is the network complex enough to be capable to encode a solution?

- Is it possible to find solution in a reasonable amount of time?

- How can we guarantee that a trained network is matching closely enough our problem domain and hidden regularities in the data?

In the recent years modern neurophysiology advanced to a big extent in understanding human brain functions and structure $[3,5,7,8,9,10]$, however, there is a lack of this knowledge application to real ANN learning algorithms. Each learning algorithm and each network topology should be carefully developed to solve more or less complex problem in real life. One may say that almost each serious application requires its own network topology, algorithm and data preprocessing.
This article presents a survey of several ways to improve ANN learning possibilities according to human brain structure and functioning, especially one example of this concept - neuroplasticity - automatic adaptation of ANN topology to the problem domain.

\section{NATURAL AND ARTIFICIAL NEURAL NETWORKS}

Human brain consists of different types of cells called neurons which are connected with each other thus making a network of neurons. This is a very simplified model because in creation and training of ANN we ignore chemical processes, frequency of neurons, features of different brain parts and many other things that are not understood or even unknown (see Fig. 1).

That is why it would be worth mentioning that all "natural" neurons and neural networks used by scientists and also adopted here in this article are very primitive and far from a real world.

Keeping in mind this limitation Figure 2 gives a "basic neuron” scheme, adopted from [1]. 


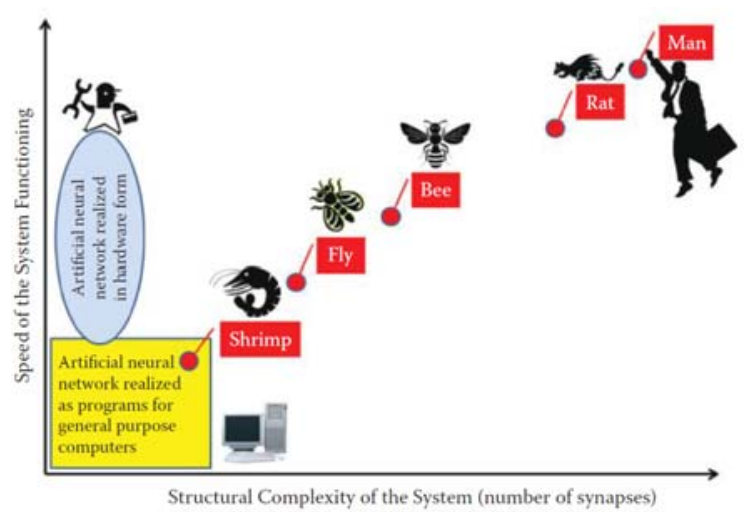

Fig. 1. ANN in comparison with real biological system considering speed of functioning and level of structural complexity [12]

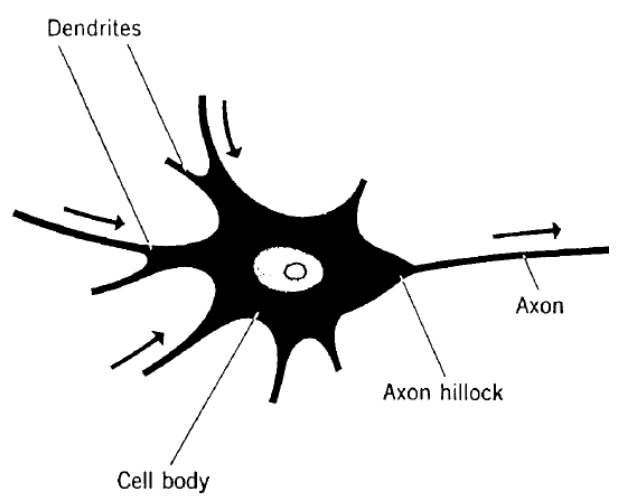

Fig. 2. Natural neuron scheme

The natural neural network (or brain) consists of billions interconnected neurons, where each neuron receives input signals from other neurons by its dendrites and produces an output to other neuron by its axon. The biological neural network complexity depends on the organism, e.g. a few hundred in some simple creatures and hundreds of billions in human brain [1].

Using this simplified biological neuron as a model on artificial neuron (software model) has been created and implemented in many types of ANN (see Fig. 3):

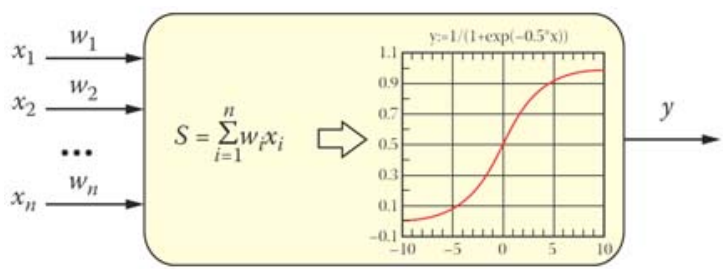

Fig. 3. The structure of widely used Multilayer Perceptron neuron (MLP) mathematical model [12]

In Fig. 3 artificial neuron receives its inputs $x_{1} \ldots x_{n}$ weighted with $\mathrm{w}_{1} \ldots \mathrm{w}_{\mathrm{n}}$ (these weights represent the strength of connection). All inputs are combined and calculated with a sum function and then propagated to activation function (sigmoid in this case). After calculations neuron gives output value y, which is fed to other neurons or is treated like a final output signal of the network.

More than hundred years ago scientists discovered several regions in the brain, each of which is responsible for its own functions (see Fig. 4). Still there is only a beginning of understanding how we are thinking and how our brain is operating. Anyway Artificial Intelligence (AI) experts are trying to simulate some functional or structural features of the brain in ANN and other AI applications. For instance, the discovery of regions in the brain is implemented by committees of networks, where several networks with different configurations are devoted to solve one task as a team. Another application of this idea is hybrid networks when a complete artificial network consists of several different parts, e.g. backpropagation and Kohonen self-organizing maps.

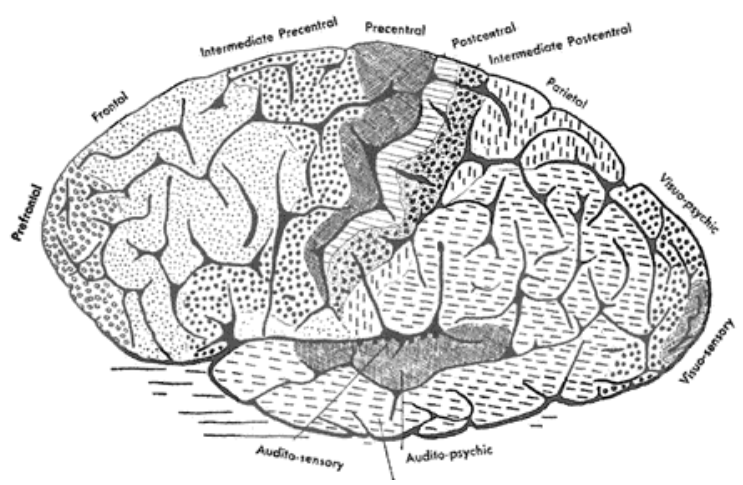

Fig.4. Anatomical parcellation of the human cerebral cortex, the left hemisphere as rendered by Alfred Campbell [9]

Figure 5 shows several examples of ANN with different structure and types. Accordingly, different learning algorithms are used to train such kind of networks: error back-propagation, self-organizing maps, recurrent learning, algorithms of hybrid types etc.

It should be noted that this article is not giving a complete review of all possible kinds of ANN, even a comprehensive book can hardly deal with this topic. The author is just showing the main principles and connection with basic brain and neurophysiology discoveries. This is an attempt to find new ways of improving existing ANN and its learning algorithms. Human brain is a wonderful instrument and we should be able to use it properly. ANN could help us in this task - modeling and solving different problems with this technology will lead us to a better understanding of our true nature. 


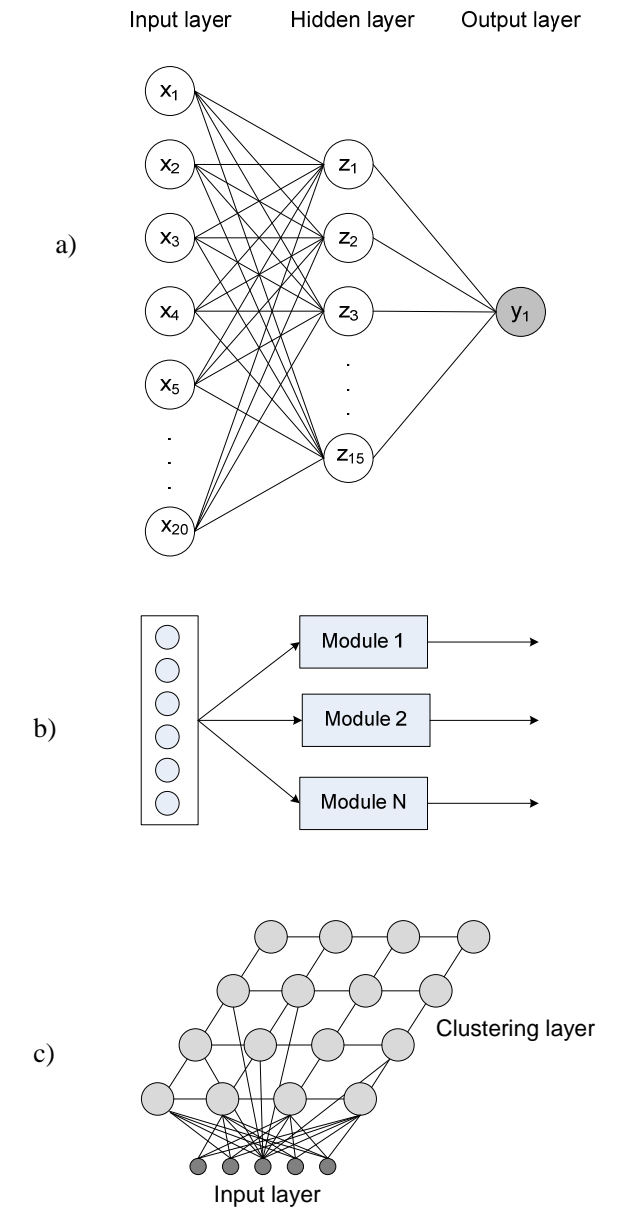

Fig. 5. ANN of different structure and learning algorithm: a) MLP with 3 layers and 36 neurons; b) committee of networks, where module 1-3 are ANN with different parameters; c) Kohonen selforganizing map.

\section{POSSIBILITIES OF IMPROVING ANN LEARNING ALGORITHMS}

When we consider an artificial neural network to be constructed and trained for solving a real problem the question of complexity arise. Human brain consists of hundreds of billions of neurons but even most powerful computer can represent only a small part of it not even taking into consideration functional properties but just number of neurons.

ANN is trained individually for each particular task and its structure and parameters depends on this learning outcome. The next problem is learning time, which is required to train a network and find a good solution of a problem. The learning time, of course, depends on effectiveness of the algorithm, implementation technology with specific software and hardware resources available. One can build a big ANN with many neurons and train it on a specific learning dataset, however, there is a problem of socalled “over-fitting”, when a network fully represents learning data remembering all these data in its weights and is failed to find hidden regularities of the task.

These are only some problems connected with ANN and some of them are more or less successfully solved. Here are some ideas of how to improve existing ANN learning algorithms according to brain theory.

The artificial neuron strength depends on its weight matrix (see Fig. 3). And the main idea of learning is to adjust these weights according to learning data. The learning time is highly dependent on initially generated weights and normalization of input data. Different normalization techniques are used to solve this issue [12].

Each real phenomenon can be fully described and understood using knowledge from many fields of science. In this case modern neurobiology could give many insights to AI scientists and especially those who are working with ANN

Human connectomics is a part of neurobiology that tries to map and analyze brain connectivity, across all scales, from the micro-level connections between neurons to macro-level between brain regions [3]. These data usually are obtained by incorporating magnetic resonance imaging (MRI) information into a form of a matrix (see Fig. 6). Then all nodes are fully described and a schematic representation of a brain region is obtained.

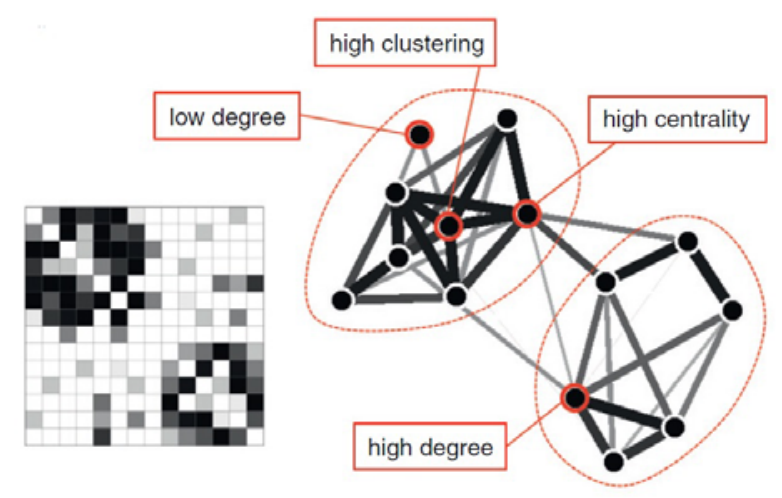

Fig.6. Schematic example of a weighted undirected connection matrix and the corresponding graph structure for a small network of 14 nodes. Nodes with high/low degree, high clustering and high centrality are indicated (adopted from [3])

When one obtains information about brain region the ANN could be designed on the base of this knowledge. In this case the ANN would be much closer to real biological neural network and probably will lead scientists to a better understanding of brain and will allow solving more complex problems than ever before.

Another very important feature of a human brain is neural plasticity or neuroplasticity [7]. This means the ability of brain to teach and to change itself according to situation. These changes include:

- Growing new neurons;

- Altering the distribution and location of neurons; 
- Modifying new synaptic connections between existing neurons in the brain regions and between these regions.

This interesting feature of human brain is partially implemented in ANN. For example, the neural network pruning techniques have been developed in the recent years $[5,13]$. The main idea is to remove unnecessary neurons from the network using certain parameter as an indicator. As a result fully connected ANN is optimized and the number of neurons is decreased thus getting smaller learning time and hardware resources (see Fig. 7).
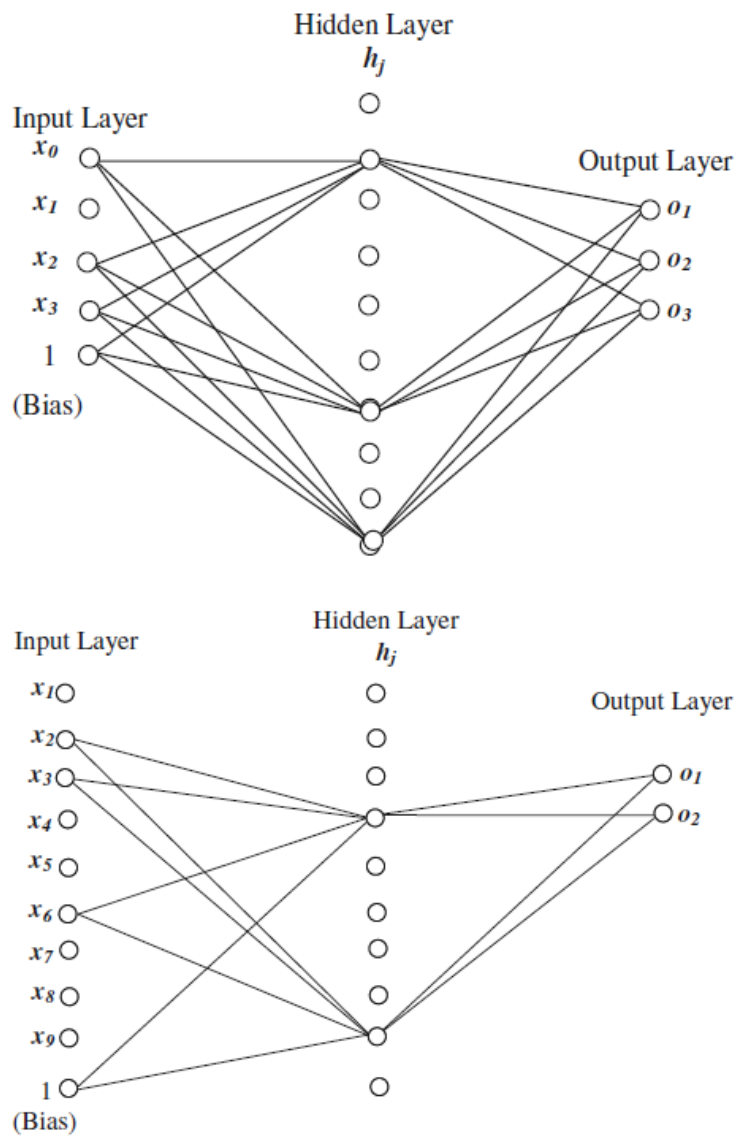

Fig. 7. Two examples of pruned networks (adopted from [5])

Figure show that in both cases the ANN is reduced by more than $50 \%$ (input and hidden layers) and is keeping its classification precision on $94 \%$ and $97 \%$ of correctly classified pattern respectively [5].

This approach is just removing unnecessary neurons and connections between them, still there may be a need for additional neurons and connections.

\section{CONCLUSIONS AND FUTURE WORK}

This article is a beginning of understanding how modern neurobiology could help artificial neural network researchers in their studies. We are just in the beginning of new complete and true science where every aspect of a whole is observed, described and fully understood.

There is a strong need for cooperation among scientists from different fields of science in order to get a better understanding who we are and where are we going. Artificial Neural Networks can help us to understand the functions of a brain from a different perspective, solving new tasks and real-world problems.

The directions of future study are:

- Development and creation of a new ANN structure based on MRI and neurobiological prototype of a human brain.

- Creation of a universal ANN learning algorithm which could optimize a neural network in a way of not only removing, but also adding necessary neurons and/or connections between neurons according to a problem solved.

- Development and implementation of a network committee model based on a brain functioning principles.

The ANN is a well-known technology and very widely developed and popular among AI researchers. However, there is always a place for new ideas and breakthrough. The author really hopes that the new development in neurobiology and ANN would come close together and help us in our everyday life.

\section{REFERENCES}

[1] M. A. Arbib, The Handbook of Brain Theory and Neural Networks. Cambridge MA: The MIT Press, 2003

[2] G. Ascoli, Computational Neuroanatomy: Principles and Methods. Totowa, New Jersey: Humana Press, 2002.

[3] T. Behrens, O. Sporns, "Human Connectomic," Current Opinion in Neurobiology, Vol. 22, pp 144-153, 2012.

[4] S. Boccaletti, V. Latora, Y. Moreno, M. Chavez, D.-U. Hwang, "Complex networks: Structure and dynamics," Physics Reports, vol. 424, pp. 175-308, Feb. 2006.

[5] M. Gethsiyal Augasta, T. Kathirvalavakumar, „A Novel Pruning Algorithm for Optimizing Feedforward Neural Network of Classification Problem," Neural Processing Letters, Vol. 34, pp 241-258, Dec. 2011.

[6] M. Kaiser, "A tutorial in connectome analysis: Topological and spatial features of brain networks," NeuroImage, Vol. 57, Issue 3, pp 892-907, Aug. 2011.

[7] Y. Perwej, F. Parwej, “A Neuroplasticity (Brain Plasticity) Approach to Use in Artificial Neural Network,” International Journal of Scientific \& Engineering Research, Vol. 3, Issue 6, pp 1-9, Jun. 2012.

[8] M. Rubinov, O. Sporns, "Complex network measures of brain connectivity: Uses and interpretations,” NeuroImage, Vol. 52, Issue 3, pp 1059-1069, Sept. 2010.

[9] O. Sporns, "From simple graphs to the connectome: Networks in neuroimaging,” NeuroImage, Vol. 62, pp 881-886, 2012.

[10] O. Sporns, Networks of the Brain. Cambridge MA: The MIT Press, 2011.

[11] C. J. Stam, E. C. W. Van Straaten, "The organization of physiological brain networks," Clinical Neurophysiology, Vol. 123, pp 1067-1087, 2012.

[12] R. Tadeusiewicz, R. Chaki, N. Chaki. Exploring Neural Networks with C\#. New York: CRC Press, 2015.

[13] X. Zeng, D. S. Yeung, "Hidden neuron pruning of multilayer perceptrons using a quantified sensitivity measure," Neurocomputing, Vol.69, pp 825-837, 2006. 